Mathematical Research Letters 5, 599-603 (1998)

\title{
SYMPLECTICALLY ASPHERICAL MANIFOLDS WITH NONTRIVIAL $\pi_{2}$
}

\author{
RoberT E. GOMPF
}

\begin{abstract}
We construct closed symplectic manifolds for which spherical classes generate arbitrarily large subspaces in 2-homology, such that the first Chern class and cohomology class of the symplectic form both vanish on all spherical classes. We construct both Kähler and non-Kähler examples, and show independence of the conditions that these two cohomology classes vanish on spherical homology. In particular, we show that the symplectic form can pair trivially with all spherical classes even when the Chern class pairs nontrivially.
\end{abstract}

Floer's original approach to the Arnol'd Conjecture $[\mathrm{F}]$ worked for closed symplectic manifolds $(X, \omega)$ satisfying the additional hypotheses that $[\omega]$ and $c_{1}(\omega)$ in $H^{2}(X ; \mathbb{R})$ should vanish on all spherical homology classes. While more recent work has avoided these hypotheses in this particular context, elsewhere the question has persisted of which symplectic manifolds satisfy the above hypotheses. Clearly the hypotheses are true for aspherical manifolds, but there are apparently no other published examples for which $[\omega]$ vanishes on all spherical classes, prompting various people to ask whether such "symplectically aspherical" manifolds are always aspherical. In this note, we answer the question in the negative. (The author has been informed that J. Kollár has also reached this conclusion see Remark 8.) To be more precise, let $\Pi(X)=\operatorname{span}_{\mathbb{R}}\left(\operatorname{Im}\left(\pi_{2}(X) \rightarrow H_{2}(X ; \mathbb{R})\right)\right)$ denote the subspace of $H_{2}(X ; \mathbb{R})$ spanned by spherical classes. We construct families of closed, symplectic 4 -manifolds $(X, \omega)$ for which $\Pi(X)$ has arbitrarily large dimension, but both $\omega$ and $c_{1}(\omega)$ vanish on $\Pi(X)$. We produce such a family for which each $\omega$ is a Kähler form, and a different family for which no $X$ is homotopy equivalent to a Kähler manifold. We also show that the conditions that $\omega$ and $c_{1}(\omega)$ vanish on $\Pi(X)$ are independent - in particular, we can have $\omega \mid \Pi(X)=0$ but $c_{1}(\omega) \mid \Pi(X) \neq 0$. Note that since $\Pi(X)=H_{2}(X ; \mathbb{R})$ when $X$ is simply connected, any closed symplectic manifold with $\omega \mid \Pi(X)=0$ must have infinite fundamental group. The question of which topologies (e.g. fundamental groups) are realized by symplectically aspherical manifolds seems highly nontrivial.

Our construction is based on symplectic branched coverings. As observed by Gromov ([G], 3.4.4(E)), symplectic structures can be lifted under branched

Received September 24, 1998.

Partially supported by NSF grant DMS-9625654. 
coverings with symplectic branch loci. The following lemma pins down the details. We denote the Poincaré duality isomorphism by $P D$.

Lemma 1. Let $(X, \omega)$ be a closed symplectic manifold with a codimension-2 symplectic submanifold $B$. Suppose that $\pi: \widetilde{X} \rightarrow X$ is a branched covering map with branch locus $B$, and the manifold $\pi^{-1}(B) \subset \widetilde{X}$ has components $B_{1}, \ldots, B_{n}$, oriented via the symplectic structure on $B$. Let $d_{i}>0$ denote the multiplicity of $\pi$ along $B_{i}$. Then there is a symplectic form $\widetilde{\omega}$ on $\widetilde{X}$ with $[\widetilde{\omega}]=\pi^{*}[\omega] \in H^{2}(\widetilde{X} ; \mathbb{R})$, and $c_{1}(\widetilde{\omega})=\pi^{*} c_{1}(\omega)+\sum\left(1-d_{i}\right) P D\left[B_{i}\right] \in H^{2}(\widetilde{X} ; \mathbb{Z})$.

Proof. The form $\pi^{*} \omega$ is symplectic on $\widetilde{X}-\bigcup_{d_{i}>2} B_{i}$. By standard symplectic topology ([MS] Example 5.10 and Theorem 3.29), we can find a closed tubular neighborhood $N$ of $B$ in $X$ with a Hamiltonian circle action, whose fixed set is $B=H^{-1}(0)$ for the Hamiltonian $H: N \rightarrow[0, \varepsilon]$. Symplectic reduction of each $H^{-1}(t)$ gives a family $\omega_{t}, t \in[0, \varepsilon]$, of symplectic forms on $B$, with $\omega_{0}=\omega \mid B$. For a suitable family $\alpha_{t}$ of 1 -forms on the total space of the circle bundle $p$ : $\partial N \rightarrow B$, the formula $p^{*} \omega_{t}+\alpha_{t} \wedge d t$ defines a symplectic form on $[0, \varepsilon] \times \partial N$, and symplectic cutting at $t=0$ recovers the original form $\omega$ on $N$ ([L], cf. also [MS] Example 5.10). Now we use $\pi$ to lift this structure to a tubular neighborhood $N_{i}$ of each component $B_{i}$ of $\pi^{-1}(B)$. The form and $S^{1}$-action on $[0, \varepsilon] \times \partial N$ lift to a form $\omega^{\prime}$ and Hamiltonian circle action on $[0, \varepsilon] \times \partial N_{i}$ with Hamiltonian $d_{i} H \circ \pi$. Since $\omega^{\prime}$ agrees with $\pi^{*} \omega$ on $(0, \varepsilon] \times \partial N_{i} \approx N_{i}-B_{i}$, symplectic cutting at 0 yields a symplectic form $\widetilde{\omega}$ on $\widetilde{X}$ that agrees with $\pi^{*} \omega$ outside of $\bigcup N_{i}$ (and with $\widetilde{\omega}=\pi^{*}(\omega \mid B)$ on each $\left.B_{i}\right)$. To check that $[\widetilde{\omega}]=\pi^{*}[\omega]$, we evaluate both on an arbitrary homology class, which we can assume is represented by a surface $F \subset \widetilde{X}$ intersecting each $N_{i}$ in a union of $S^{1}$-invariant normal disks. For each such disk $D$, it is easy to see that $\int_{D} \widetilde{\omega}=d_{i} \int_{\pi(D)} \omega$, so since $\widetilde{\omega}=\pi^{*} \omega$ on $\widetilde{X}-\bigcup$ int $N_{i}$, we have $\langle\widetilde{\omega},[F]\rangle=\left\langle\omega, \pi_{*}[F]\right\rangle=\left\langle\pi^{*} \omega,[F]\right\rangle$, as required.

To compute $c_{1}(\widetilde{\omega})$, fix an almost-complex structure $J$ on $X$ tamed by $\omega$, with $B$ and the fibers of $N J$-holomorphic. Then $J$ lifts to a $C^{0}$ almost-complex structure $\widetilde{J}$ on $\widetilde{X}$ tamed by $\widetilde{\omega}$. Now $c_{1}(\omega)=c_{1}(J)$ is Poincaré dual to the zero locus of a generic section of $\Lambda T X$, where $\Lambda$ denotes the complex top exterior power. On $N$, we have $T X=T B \oplus \nu B$ and $\Lambda T X=\Lambda T B \otimes \nu B$. Let $\sigma$ : $B \rightarrow \Lambda T B$ be a generic section, and let $\tau: N \rightarrow \nu B$ be given by the Tubular Neighborhood Theorem (so $\tau^{-1}(0)=B$ ). Extend the section $\sigma \otimes \tau$ of $\Lambda T X \mid N$ generically over all of $X$. The resulting zero locus exhibits $c_{1}(J)$ as $P D[C]+$ $P D[B]$, for some codimension-2 submanifold $C$ of $X$ intersecting $B$ transversely. Since $\tau$ lifts to a continuous map $N_{i} \rightarrow \nu B_{i}$ vanishing with multiplicity 1 on $B_{i}$, the corresponding section of $\Lambda T \widetilde{X}$ exhibits $c_{1}(\widetilde{\omega})=c_{1}(\widetilde{J})$, as $\pi^{*} P D[C]+$ $\sum P D\left[B_{i}\right]=\pi^{*}\left(c_{1}(J)-P D[B]\right)+\sum P D\left[B_{i}\right]=\pi^{*} c_{1}(J)+\sum\left(1-d_{i}\right) P D\left[B_{i}\right]$.

Example 2. Let $X=F_{1} \times F_{2}$ be a product of two surfaces of nonzero genus, with a product symplectic form $\omega$. Fix integers $m_{1}, m_{2} \geq 1$, and $d \geq 2$. Choose $m_{1} d$ surfaces in $X$ of the form $\{p\} \times F_{2}$ and $m_{2} d$ surfaces $F_{1} \times\{q\}$, and let 
$B^{*}$ denote their union. Let $B \subset X$ be the smooth symplectic surface obtained by smoothing the $m_{1} m_{2} d^{2}$ positive double points of $B^{*}$ via the local model $\left\{z_{1} z_{2}=0\right\} \mapsto\left\{z_{1} z_{2}=\varepsilon\right\}$. Let $\pi: \widetilde{X} \rightarrow X$ be the $d$-fold cyclic branched covering of $X$, branched along $B$ with multiplicity $d$ on $\pi^{-1}(B)$. (There is only one such covering, up to diffeomorphisms of $X$.) The following two theorems immediately show that $\operatorname{dim} \Pi(\widetilde{X}) \geq m_{1} m_{2} d^{2}(d-1)$, but the form $\widetilde{\omega}$ given by Lemma 1 satisfies $\widetilde{\omega}\left|\Pi(\widetilde{X})=c_{1}(\widetilde{\omega})\right| \Pi(\widetilde{X})=0$.

Theorem 3. For $(\widetilde{X}, \widetilde{\omega})$ as in Lemma 1, if $\omega \mid \Pi(X)=0$, (e.g., if $\pi_{2}(X)=0$,) then $\widetilde{\omega} \mid \Pi(\widetilde{X})=0$. If $\pi_{2}(X)=0$ and $\pi^{-1}(B)$ is connected, then $c_{1}(\widetilde{\omega}) \mid \Pi(\widetilde{X})=0$.

Proof. If $\alpha \in H_{2}(\widetilde{X} ; \mathbb{Z})$ is represented by a map of a sphere, then so is $\pi_{*} \alpha \in$ $H_{2}(X ; \mathbb{Z})$. Thus $\langle[\widetilde{\omega}], \alpha\rangle=\left\langle[\omega], \pi_{*} \alpha\right\rangle=0$. If, in addition, $\pi_{2}(X)=0$, then $\pi_{*} \alpha=0$, so the intersection number $\pi_{*} \alpha \cdot B$ vanishes. Connectedness of $\pi^{-1}(B)$ now implies that $\alpha \cdot \pi^{-1}(B)=0$ and $\left\langle c_{1}(\widetilde{\omega}), \alpha\right\rangle=\left\langle c_{1}(\omega), \pi_{*} \alpha\right\rangle=0$.

Theorem 4. Let $\pi: \widetilde{X} \rightarrow X$ be a d-fold branched covering of orientable 4manifolds, whose branch locus $B \subset X$ is obtained from a generically immersed surface $B^{*} \subset X$ by smoothing all double points as in Example 2 (up to orientation). If $B^{*}$ has $k \geq 1$ double points, then $\Pi(\tilde{X}) \neq 0$. If $\pi$ is injective on $\pi^{-1}(B)$, then $\operatorname{dim} \Pi(\widetilde{X}) \geq k(d-1)$.

Proof. At each double point of $B^{*}$, a 4-ball neighborhood $K$ intersects $B$ in the complex curve $z_{1} z_{2}=\varepsilon$, which is the Milnor fiber $M(2,2)$. Each component of $\pi^{-1}(K)$ intersects one $B_{i}$, and is the $d_{i}$-fold cover of $K$ branched along $K \cap B$. This is diffeomorphic to the Milnor fiber $M\left(2,2, d_{i}\right)$, which is the plumbing along a linear graph of $d_{i}-1$ spheres of square -2 . The intersection matrix of these $d_{i}-1$ spheres has nonzero determinant, so each component of each $\pi^{-1}(K)$ contributes $d_{i}-1$ spheres to a linearly independent subset of $\Pi(\widetilde{X})$. If $\pi \mid \pi^{-1}(B)$ is injective, then each $d_{i}=d$, so we obtain $\operatorname{dim} \Pi(\widetilde{X}) \geq k(d-1)$ (and in general, $\operatorname{dim} \Pi(\widetilde{X}) \geq k d-\ell$, where $\ell$ is the number of components of $\left.\bigcup \pi^{-1}(K)\right)$.

Example 5 - Kähler manifolds. We find a family as in Example 2 with $\Pi(\widetilde{X})$ arbitrarily large, for which each $\widetilde{\omega}$ is a Kähler form. Suppose that each $F_{i}$ is given as a complex curve of degree $m_{i}$ in $\mathbb{C P}^{N_{i}}$. Let $H_{i} \subset \mathbb{C P}^{N_{i}}$ be a generic degree- $d$ hypersurface, and choose the $m_{1} d$ points $p$ in Example 2 to be the set $F_{1} \cap H_{1}$. Similarly, choose the points $q$ to be $F_{2} \cap H_{2}$. Thus, each of these finite subsets of $F_{i}$ is exhibited as the zero locus of a holomorphic section of $L_{i}^{\otimes d}$, where $L_{i} \rightarrow F_{i}$ is the restriction of the hyperplane bundle of $\mathbb{C P}^{N_{i}}$. The corresponding zero locus of $L_{1}^{\otimes d} \otimes L_{2}^{\otimes d} \rightarrow X=F_{1} \times F_{2}$ is the singular surface $B^{*}$. By varying our choices of $H_{i}$, it is easy to find three holomorphic sections of $L_{1}^{\otimes d} \otimes L_{2}^{\otimes d}$ whose common zero locus is empty - thus, the complete linear system of holomorphic sections of $L_{1}^{\otimes d} \otimes L_{2}^{\otimes d}$ has empty base locus. By Bertini's Theorem, a generic holomorphic section $\rho$ of $L_{1}^{\otimes d} \otimes L_{2}^{\otimes d}$ has smooth zero locus, so the required perturbation of $B^{*}$ to a smooth surface $B=\rho^{-1}(0)$ can be done holomorphically. To find a Kähler form $\widetilde{\omega}$ on the resulting $\widetilde{X}$ 
satisfying the conclusion of Lemma 1 , note that the $d$-fold tensor power map $p: L_{1} \otimes L_{2} \rightarrow\left(L_{1} \otimes L_{2}\right)^{d}=L_{1}^{\otimes d} \otimes L_{2}^{\otimes d}$ is a $d$-fold covering map branched along the zero section. Since the section $\rho: X \rightarrow L_{1}^{\otimes d} \otimes L_{2}^{\otimes d}$ is a diffeomorphism onto its image, we can identify $\widetilde{X}$ with $p^{-1}(\operatorname{Im} \rho) \subset L_{1} \otimes L_{2}$ and $\pi: \widetilde{X} \rightarrow X$ with the restriction of projection $L_{1} \otimes L_{2} \rightarrow X$. If we identify $X$ with the zero section of $L_{1} \otimes L_{2}$, then any Kähler form $\Omega$ on the total space of $L_{1} \otimes L_{2}$ restricts to Kähler forms $\widetilde{\omega}$ on $\widetilde{X}$ and $\omega$ on $X$. For any $\alpha \in H_{2}(\widetilde{X} ; \mathbb{R})$, the classes $\alpha$ and $\pi_{*} \alpha$ have the same image in $H_{2}\left(L_{1} \otimes L_{2} ; \mathbb{R}\right)$, so $\langle\widetilde{\omega}, \alpha\rangle=\left\langle\Omega, \pi_{*} \alpha\right\rangle=\left\langle\omega, \pi_{*} \alpha\right\rangle$, implying that $[\widetilde{\omega}]=\pi^{*}[\omega]$ as required. The Chern class formula follows as before (and is well-known since $\pi$ is holomorphic), so we again have $\widetilde{\omega}\left|\Pi(\widetilde{X})=c_{1}(\widetilde{\omega})\right| \Pi(\widetilde{X})=0$.

Example 6 - Non-Kähler manifolds. Let $(X, \omega)$ denote the KodairaThurston manifold, obtained as the quotient of $\left(\mathbb{R}^{4}, d x_{1} \wedge d x_{2}+d x_{3} \wedge d x_{4}\right)$ by the discrete group of symplectomorphisms generated by unit translations in the $x_{1}, x_{2}$ and $x_{3}$ directions together with $\left(x_{1}, x_{2}, x_{3}, x_{4}\right) \mapsto\left(x_{1}+x_{2}, x_{2}, x_{3}, x_{4}+1\right)$. The projection $\left(x_{1}, x_{2}, x_{3}, x_{4}\right) \mapsto\left(x_{3}, x_{4}\right)$ induces a bundle structure $p: X \rightarrow T^{2}$ with torus fibers and a section $x_{1}=x_{2}=0$ with trivial normal bundle. As in Example 2, choose $m_{1} d$ fibers and $m_{2} d$ parallel copies of the section, smooth the intersections of the resulting $B^{*}$ to obtain a smooth symplectic surface $B \subset X$, and let $\pi: \widetilde{X} \rightarrow X$ be the resulting $d$-fold branched cover. As before, we obtain examples with $\Pi(\widetilde{X})$ arbitrarily large, but with the form $\widetilde{\omega}$ of Lemma 1 satisfying $\widetilde{\omega}\left|\Pi(\widetilde{X})=c_{1}(\widetilde{\omega})\right| \Pi(\widetilde{X})=0$. This time, however, no $\widetilde{X}$ has the homotopy type of a Kähler manifold, as we verify by showing that $b_{1}(\widetilde{X})=3$. Recall that $b_{1}(X)=3$, since $H_{1}(X ; \mathbb{Z})$ has 4 canonical generators $\alpha_{1}, \ldots, \alpha_{4}$ with $\alpha_{i}$ descending from the $x_{i}$-axis, and the monodromy introduces the relation $\alpha_{2}=\alpha_{1}+\alpha_{2}$. Since $\pi_{*}: H_{1}(\widetilde{X} ; \mathbb{Z}) \rightarrow H_{1}(X ; \mathbb{Z})$ is surjective, we have $b_{1}(\widetilde{X}) \geq 3$. The Milnor fibers $M(2,2, d) \subset \widetilde{X}$ arising as in the proof of Theorem 4 are simply connected, so we can collapse these to points, obtaining a singular space $\widetilde{X}^{*}$ with $b_{1}\left(\widetilde{X}^{*}\right)=b_{1}(\widetilde{X})$ and a map $\pi^{*}: \widetilde{X}^{*} \rightarrow X$ that is a $d$-fold covering branched along $B^{*}$. The composite $p \circ \pi^{*}: \widetilde{X}^{*} \rightarrow T^{2}$ is a singular fibration whose $m_{1} d$ singular fibers are tori. By transversality, $H_{1}\left(\widetilde{X}^{*} ; \mathbb{R}\right)$ is generated by cycles disjoint from the singular fibers, hence, by cycles in a regular fiber together with a pair of classes lifted from the base $T^{2}$. (Meridians to the critical values in $T^{2}$ lift to nullhomologous $\mathbb{R}$-cycles in $\widetilde{X}^{*}$.) Since any cycle in a regular fiber is homologous to one in a preassigned singular fiber, $H_{1}\left(\widetilde{X}^{*} ; \mathbb{R}\right)$ is generated by 4 classes $\tilde{\alpha}_{1}, \ldots, \tilde{\alpha}_{4}$ lifted from cycles in $B^{*}$ representing $\alpha_{1}, \ldots, \alpha_{4}$. Since the relation $\alpha_{2}=\alpha_{1}+\alpha_{2}$ is represented by a surface in $X$ disjoint from $B^{*}$ and lifting trivially to $\widetilde{X}^{*}$, we obtain the relation $\widetilde{\alpha}_{2}=\widetilde{\alpha}_{1}+\widetilde{\alpha}_{2}$ in $H_{1}\left(\widetilde{X}^{*} ; \mathbb{R}\right)$, so $b_{1}(\widetilde{X})=b_{1}\left(\widetilde{X}^{*}\right)=3$ as required.

Theorem 7. The conditions $\omega \mid \Pi(X)=0$ and $c_{1}(\omega) \mid \Pi(X)=0$ are independent.

Proof. We have shown that both $\omega$ and $c_{1}(\omega)$ can vanish on a nontrivial $\Pi(X)$. A small perturbation of $\omega$ gives examples with $\omega\left|\Pi(X) \neq 0, c_{1}(\omega)\right| \Pi(X)=0$. (The $K 3$-surface is another such example.) Any simply-connected Kähler surface 
other than $K 3$ satisfies $\omega, c_{1}(\omega) \neq 0$ on $\Pi(X)=H_{2}(X ; \mathbb{R})$. For the remaining case $\omega\left|\Pi(X)=0, c_{1}(\omega)\right| \Pi(X) \neq 0$, begin with the torus $T^{4}=\mathbb{C}^{2} / \mathbb{Z}^{4}$ with the standard Kähler form $\omega$. Let $B^{*} \subset T^{4}$ be the union of tori descending from the 4 lines $z_{i}= \pm \frac{1}{4}$ in $\mathbb{C}^{2}$, and let $B$ be the symplectic surface obtained by smoothing the 4 intersections $\left( \pm \frac{1}{4}, \pm \frac{1}{4}\right)$ of $B^{*}$ using the local model $z_{1} z_{2}=-\varepsilon, 0<\varepsilon \ll 1$ (up to translation and perturbation away from the double points). The torus $T \subset T^{4}$ determined by the equation $z_{2}=\bar{z}_{1}$ intersects $B^{*}$ in the 2 points $\pm\left(\frac{1}{4}, \frac{1}{4}\right)$ and is disjoint from $B$. Since $T$ is Lagrangian, it is easy to perturb $\omega$ so that $T$ becomes symplectic. Let $(\widetilde{X}, \widetilde{\omega})$ be a 2 -fold cover of $T^{4}$ branched along $B$ (and chosen to be trivial over $T$ ), and let $\widetilde{T}$ be one of the 2 lifts of $T$ to $\widetilde{X}$. The vanishing cycle of $B$ created by smoothing $B^{*}$ at $\left(\frac{1}{4}, \frac{1}{4}\right)$ bounds a disk $D$ in $T^{4}$ intersecting $T$ transversely in one point. (The coordinate change $z_{1}=u_{1}+i u_{2}$, $z_{2}=-u_{1}+i u_{2}$ (following the appropriate translation) exhibits $B$ as $u_{1}^{2}+u_{2}^{2}=\varepsilon$, $D$ as the $\sqrt{\varepsilon}$-disk in $\mathbb{R}^{2}$, and $T$ as $i \mathbb{R}^{2}$.) The 2 lifts of $D$ to $\tilde{X}$ fit together to form a sphere $S \subset \widetilde{X}$ intersecting $\widetilde{T}$ transversely in a single point. Let $(\widehat{X}, \widehat{\omega})$ be any branched cover of $\widetilde{X}$ with branch locus given by 2 parallel copies of $\widetilde{T}$. (Such a branched cover exists since $\widetilde{T}$ is symplectic with trivial normal bundle - e.g., send the two meridians to opposite generators of $\mathbb{Z} / d$.) The sphere $S \subset \widetilde{X}$ lifts to a sphere $\widehat{S} \subset \widehat{X}$ intersecting two components $B_{i}$ of the lifted branch locus of $\widehat{X} \rightarrow \widetilde{X}$ each in one point. The two components $B_{i}$ have the same multiplicity $d \geq 2$, and the signs $B_{i} \cdot \widehat{S}$ agree if each $B_{i}$ is symplectically oriented. Now Theorem 3 implies that $\widehat{\omega} \mid \Pi(\widehat{X})=0$ and $c_{1}(\widetilde{\omega}) \mid \Pi(\widetilde{X})=0$, so by Lemma 1 applied to $\widehat{X} \rightarrow \widetilde{X}$, we have $\left\langle c_{1}(\widehat{\omega}), \widehat{S}\right\rangle=\sum\left(1-d_{i}\right) B_{i} \cdot \widehat{S}=2(1-d) \neq 0$.

Remark 8. J. Kollár produced a different construction of symplectically aspherical Kähler manifolds with $\Pi(X) \neq 0$, which preceded the author's work by several months. He now observes that both constructions are based on a common idea: If $(X, \omega)$ is symplectic, $f: X \rightarrow Y$ is a continuous map to a topological space with $\pi_{2}(Y)=0$, and $[\omega]=f^{*} \Omega$ for some $\Omega \in H^{2}(Y ; \mathbb{R})$, then $\omega \mid \Pi(X)=0$. (The proof is the same as for Theorem 3.) In our case, $f$ is a branched covering, whereas Kollár took $f$ to be an inclusion of algebraic varieties with $Y$ a $K(\pi, 1)$ (e.g., an Abelian variety) with $\operatorname{dim}_{\mathbb{C}} Y \geq 3$.

\section{References}

[F] A. Floer, Symplectic fixed points and holomorphic spheres, Commun. Math. Phys. 120 (1989), 575-611.

[G] M. Gromov, Partial Differential Relations, Ergebnisse der Mathematik und ihrer Grenzgebiete (3) [Results in Mathematics and Related Areas (3)], 9, Springer-Verlag, BerlinNew York, 1986.

[L] E. Lerman, Symplectic cuts, Math. Res. Lett. 2 (1995), 247-258.

[MS] D. McDuff and D. Salamon, Introduction to symplectic topology, Oxford Math. Monographs, Clarendon Press, NY, 1995.

Department of Mathematics, University of Texas at Austin, Austin TX 78712

E-mail address: gompf@math.utexas.edu 